VII JORNADAS DE DIFUSIÓN DE LA INVESTIGACIÓN Y EXTENSIÓN - FCV-UNL

RESUMEN EXTENDIDO

\title{
DETERMINACIÓN DE LA CONCENTRACIÓN EFECTIVA 50, 90 Y 99 DE IVERMECTINA SOBRE UNA CEPA DE GARRAPATA COMÚN DEL BOVINO (Rhipicephalus (Boophilus) microplus) PROVENIENTE DE LA PROVINCIA DE SANTA FE-ARGENTINA
}

\author{
Torrents $\mathrm{J}^{1}$, Formentini $\mathrm{E}^{2}$, Nava $\mathrm{S}^{3}$ \\ ${ }^{1}$ Cátedra de Zoología, FCV-UNL \\ ${ }^{2}$ Laboratorio de Farmacología y Toxicología, FCV-UNL \\ ${ }^{3}$ Instituto Nacional de Tecnología Agropecuaria (INTA EEA Rafaela) \\ *Correspondencia: Torrents J., E-mail: torrentsjorgelina@gmail.com
}

Editado por: R. Sobrero, V. Matiller, C. Baravalle

DETERMINATION OF THE EFFECTIVE CONCENTRATION 50, 90 AND 99 OF IVERMECTIN AGAINST A STRAIN OF

\section{SUMMARY}

The aim of this study was to obtain the sensitivity data of the native strains of $R$. microplus to ivermectin (IVM) in the province of Santa Fe-Argentina, in order to detect the emergence of resistant strains. Native (field isolation) and susceptible (reference) larvae of $R$. microplus were treated with increasing concentrations of IVM in order to determine its efficacy at different doses. The statistical treatment of the concentration-efficacy curves was performed by the PROBIT model, who is a standardized method proposed by FAO. The results obtained indicated that the larvae isolated were susceptible, being the values of LD50 (9.66 ppm) and LD90 (17.41 ppm), which were lower than those of the reference strain (LD50:13.58 ppm, LD90:19.31 ppm), and the RR (resistance ratio) were less than one (RR50: 0.711; RR90: 0.901). The DL99 (51.48 ppm) was only obtained in the reference strain to determine the discriminant dose (DL99x2), which is a parameter that could be used to easily discriminate susceptible and resistant larvae populations.

Palabras clave: Rhipicephalus microplus, test de inmersión de larvas, ivermectina, control de garrapatas.

Keywords: Rhipicephalus microplus, larval immersion test, ivermectin, tick control.

Rhipicephalus (Boophilus) microplus (garrapata común del bovino) es el ectoparásito hematófago de los bovinos de mayor importancia a nivel mundial para la producción pecuaria. En Argentina esta garrapata se encuentra distribuida en la zona norte al paralelo $30 \circ-310 \mathrm{~S}$, generando grandes pérdidas directas e indirectas en la producción pecuaria, además de ser el vector exclusivo de Babesia bigemina y $B$. bovis. El método más utilizado en la Argentina para controlar a este ectoparásito es el empleo de químicos, cuyo uso indiscriminado ha derivado en la emergencia de resistencia a los siguientes principios activos: arsenicales, organofosforados, piretroides, formamidinas y los fenilpirazoles. Es posible que la resistencia ya se encuentre establecida para drogas como ivermectina (IVM), fluazurón y fipronil. El diagnostico in vitro de sensibilidad de R. microplus a IVM es una herramienta útil para conocer el nivel actual de resistencia a esta droga, además de permitir en base a los resultados, elaborar estrategias de tratamiento en función de los principios químicos que aún son activos en cada establecimiento. Para la detección de resistencia de IVM en larvas de $R$. microplus, la FAO recomienda la utilización del test de paquete de larvas (LPT) (FAO, 2004). Sin embargo, para IVM se ha demostrado que el test de inmersión de larvas (TLI) presenta mayor sensibilidad (Klafke et al., 2006). El tratamiento 
estadístico de los resultados obtenidos por estas técnicas se encuentra estandarizado por la FAO (FAO, 2004), que recomienda el uso del modelo PROBIT para el ajuste de las curvas concentración-eficacia. Es de suma importancia disponer de datos de sensibilidad de las cepas autóctonas de $R$. microplus a IVM en la región para poder detectar la emergencia de cepas resistentes, ya que no se pueden tomar como referencia resultados obtenidos en otras regiones del mundo, debido a que variaciones entre las muestras, entre los bioensayos o bien variaciones naturales, pueden determinar que los resultados de una región no sean comparables con los de otra (Jonsson et al,. 2007). El objetivo de este estudio preliminar fue determinar mediante la técnica de TLI la sensibilidad de una cepa autóctona de $R$. microplus aislada en la provincia de Santa Fe - Argentina, susceptible a IVM, a fin de disponer de parámetros que nos permitan detectar poblaciones de R. microplus susceptibles o resistentes a través del análisis de los resultados mediante el modelo PROBIT. Se utilizaron dos cepas autóctonas de $R$. microplus; una cepa sensible (0.17) identificada como S y una cepa test (0.29) identificada como $\mathrm{T}$. El ensayo de TLI se realizó según metodología reportada previamente (Klafke et al., 2006), utilizando un estándar de IVM de pureza conocida (Sigma-Aldrich). Brevemente, las larvas de R. microplus se enfrentaron a concentraciones crecientes de IVM y luego de $24 \mathrm{~h}$ de exposición se determinó la eficacia expresándola como \% de mortalidad de las mismas mediante el recuento de larvas vivas y muertas. Los resultados fueron ajustados con el modelo PROBIT (LeOra Software, 2003). Para las dos cepas (S y T) se estimaron los valores de las concentraciones efectivas $50 \%$ (C50) y $90 \%$ (C90) respectivamente, mientras que para la cepa $S$ (0.17) se estimó el valor de la concentración efectiva 99\% (C99) con la que se calculó la dosis discriminante (DD) como C99 × 2 (Jonsson et al., 2007). Para evaluar la susceptibilidad de la cepa $T$ (0.29) se utilizaron los valores de relación de resistencia (RR) que se estimaron como el cociente entre los valores de C50 de las cepas T y S (C50-T/C50-S) y entre las C90 entre las cepas $\mathrm{T}$ y $\mathrm{S}$ (C90-T/C90-S). El valor de RR permite clasificar las poblaciones de larvas en tres categorías: (i) susceptibles ( $R R \leq 1$ ); (ii) incipientemente resistentes ( $R R$ entre 1 y 2) y (iii) resistentes ( $R R>2$ ) (Robertson et al., 2007). El ajuste de las curvas de concentración (ppm) de IVM vs. \% de eficacia sobre larvas de las cepas S (0.17) y $\mathrm{T}$ (0.29) de $R$. microplus con el modelo PROBIT se presentan en la figura 1.

Los valores de C50, C90 de IVM (ppm) estimados para las cepas $S(0.17)$ y $T$ (0.29), y los valores de C99 y DD de IVM $(\mathrm{ppm})$ para la cepa $S(0.17)$ y los valores estimados de $R R$ para C50 (C50-T/C50-S) y C90 (C90-T/C90-S) se presentan en la tabla 1

Los resultados obtenidos con modelo PROBIT (LeOra Software, 2003) nos permiten inferir que la cepa T (0.29) es susceptible respecto de los parámetros de eficacia obtenidos a partir de la cepa $S(0.17)$. En la tabla 1 se puede observar que tanto los valores de C50 como de
C90 de la cepa T (0.29) son menores a los de la cepa $S$ (0.17), de manera que fue más fácil determinar la susceptibilidad de esta por medio de la estimación de RR, siendo estos valores < a 1 tanto para C50 (RR: 0,711) y C90 (RR: 0,901), lo cual se interpreta como susceptibilidad de la cepa T (0.29). En la figura 1 B puede observarse que para la cepa T (0.29), la eficacia o mortalidad del $100 \%$ de las larvas de $R$. microplus se observó a una concentración de IVM de 34,3 ppm, que es una concentración menor a la DD estimada a partir de la cepa $S(0.17)$ de 51,48 ppm (Tabla 1). En este estudio preliminar hemos corroborado in vitro la sensibilidad de nuestra cepa de referencia $S$ (0.17) que fuera previamente determinada (in vivo) por nuestro equipo de trabajo en ensayos a campo en los que se observó que la sensibilidad de esta a IVM fue $\geq$ al $95 \%$, por lo que podríamos inferir que los resultados obtenidos en los ensayos in vitro e in vivo se correlacionan. Concluyendo, en base a la determinación de la sensibilidad de nuestra cepa de referencia $S(0.17)$ hemos determinado in vitro la susceptibilidad de la cepa T (0.29). Sin embargo, este resultado también debería ser contrastado con pruebas de campo. Sería de mucha utilidad la implementación de las técnicas in vitro y a campo (in vivo) sobre cepas incipientemente resistentes y resistentes.
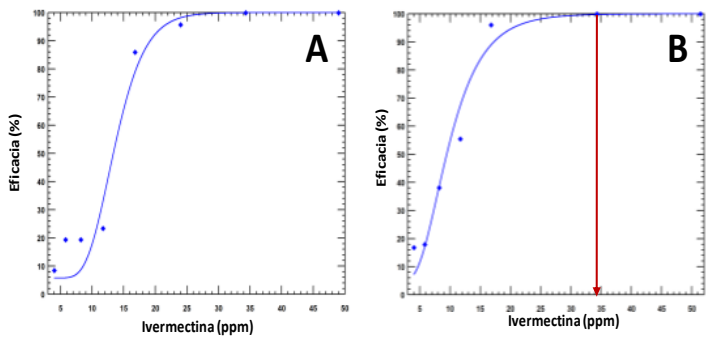

Figura 1. Ajuste de la relación concentración (ppm) y eficacia (\%) de ivermectina con el modelo PROBIT sobre larvas de $R$. microplus; A corresponde a la cepa S (0.17) y B corresponde a la cepa $T$ (0.29). En B la flecha indica la concentración de ivermectina $(34,3 \mathrm{ppm})$ con la que se observó el $100 \%$ de eficacia.

Tabla 1. Valores de C50, C90 de IVM estimados para las cepas S (0.17) y T (0.29), y los valores de C99 y dosis discriminante (DD) de IVM (ppm) para la cepa S (0.17) y los valores estimados de RR para C50 (C50-T/C50-S) y C90 (C90-T/C90-S).

\begin{tabular}{l|ccc|}
\hline Parámetros & Cepa 0.17 & Cepa 0.29 & RR \\
\hline C $_{50}(\mathrm{ppm})$ & 13,58 & 9,66 & 0,711 \\
$\mathrm{C}_{10}(\mathrm{ppm})$ & 19,31 & 17,41 & 0,901 \\
$\mathrm{C}_{99}(\mathrm{ppm})$ & 25,74 & 28,15 & - \\
DD $(\mathrm{ppm})$ & 51,48 & - & - \\
\hline
\end{tabular}

\section{Bibliografía}

FAO. 2004. Guideline resistance management and integrated parasite control in ruminants. Acaricide resistance: diagnosis, management and prevention. Agr. 
Dept. Animal Production and Health Division. Roma, Italia. 25-77 pp.

Jonsson NN, Miller RJ, Robertson JL. 2007. Critical evaluation of the modified-adult immersion test with discriminating dose bioassay for Boophilus microplus using American and Australian isolates. Vet. Parasitol. 146: 307315.

Klafke GM, Sabatini GA, de Albuquerque TA, Martins JR, Kemp DH, Miller RJ, Schumaker TT. 2006. Larval immersion tests with ivermectin in populations of the cattle tick Rhipicephalus(Boophilus) microplus (Acari: Ixodidae) from State of Sao Paulo, Brazil. Vet. Parasitol. 142: 386-390.

Robertson JL, Russell RM, Presiler HK, Savin NE. (2007). Quarantine statistics. In: Bioassays with Arthropods, 2nd ed. CRC Press. New York. 